\title{
Phenotypes and genotypes in outbred and inbred Primary microcephaly: high incidence of epilepsy
}

Sarah Duerinckx ${ }^{1}$, Julie Désir ${ }^{2}$, Camille Perazzolo ${ }^{1}$, Cindy Badoer ${ }^{3}$, Valérie Jacquemin ${ }^{1}$, Julie Soblet ${ }^{3}$, Isabelle Maystadt ${ }^{4}$, Yusuf Tunca ${ }^{5}$, Bettina Blaumeiser ${ }^{6}$, Berten Ceulemans ${ }^{6}$, Winnie Courtens ${ }^{7}$, François-Guillaume Debray ${ }^{7}$, Anne Destree $^{2}$, Koen Devriendt ${ }^{8}$, Anna Jansen $^{9}$, Kathelijn Keymolen ${ }^{9}$, Damien Lederer ${ }^{2}$, Bart Loeys ${ }^{6}$, Gert Matthijs ${ }^{8}$, Marije Meuwissen $^{6}$, Stéphanie Moortgat ${ }^{4}$, Geert Mortier ${ }^{6}$, Marie-Cécile Nassogne ${ }^{10}$, Tayeb Sekhara $^{11}$, Rudy van Coster ${ }^{12}$, Jenneke Van den Ende ${ }^{6}$, Nathalie Van der Aa ${ }^{6}$, Hilde Van Esch $^{8}$, Olivier Vanakker ${ }^{12}$, Helene Verhelst ${ }^{12}$, Catheline Vilain ${ }^{13}$, Sarah Weckhuysen ${ }^{6}$, Sandrine PASSEMARD ${ }^{14}$, alain verloes ${ }^{14}$, Alec Aeby ${ }^{13}$, Nicolas Deconinck ${ }^{13}$, Patrick Van Bogaert $^{15}$, Isabelle Pirson ${ }^{1}$, and Marc Abramowicz ${ }^{16}$

${ }^{1}$ Université Libre de Bruxelles

${ }^{2}$ Institut de Pathologie et de Génétique asbl

${ }^{3}$ Hôpital Erasme

${ }^{4}$ Institut de Pathologie et de Genetique asbl

${ }^{5}$ University of Health Sciences Turkey, Gülhane Faculty of Medicine \& Gülhane Training and Research Hospital

${ }^{6}$ University Hospital Antwerp

${ }^{7}$ Centre hospitalier universitaire de Liège

${ }^{8} \mathrm{KU}$ Leuven University Hospitals Leuven

${ }^{9}$ Universitair Ziekenhuis Brussel

${ }^{10}$ Cliniques universitaires Saint-Luc

${ }^{11}$ CHIREC

${ }^{12}$ Universitair Ziekenhuis Gent

${ }^{13}$ Hôpital Universitaire des Enfants Reine Fabiola

${ }^{14} \mathrm{APHP}$

${ }^{15} \mathrm{CHU}$ Angers

${ }^{16}$ University of Geneva

January 4, 2021

\begin{abstract}
Primary microcephaly (PM) is defined as a significant reduction in occipito-frontal circumference (OFC) of prenatal onset. Clinical and genetic heterogeneity of PM represents a diagnostic challenge. We performed detailed phenotypic and genomic analyses in a large cohort $(\mathrm{n}=169)$ of patients referred for PM, and could establish a molecular diagnosis in 38 patients. Pathogenic variants in ASPM and WDR62 were the most frequent causes in non-consanguineous patients in our cohort. In consanguineous patients, microarray and targeted gene panel analyses reached a diagnostic yield of $67 \%$, which contrasts with a much lower rate in outbred patients $(9 \%)$. Our series includes 15 previously unreported families and 11 novel pathogenic variants, and we identify novel candidate genes including IGF2BP3, DNAH2, and TSR1. We confirm progression of microcephaly over
\end{abstract}


time in affected children. Epilepsy was an important associated feature in our PM cohort, affecting $34 \%$ of patients, with various degrees of severity and seizure types. Our findings will help to prioritize genomic investigations, accelerate molecular diagnoses and improve management of PM patients.

\section{Hosted file}

PM cohort_201216_HuMu.pdf available at https://authorea.com/users/387178/articles/502428phenotypes-and-genotypes-in-outbred-and-inbred-primary-microcephaly-high-incidence-ofepilepsy
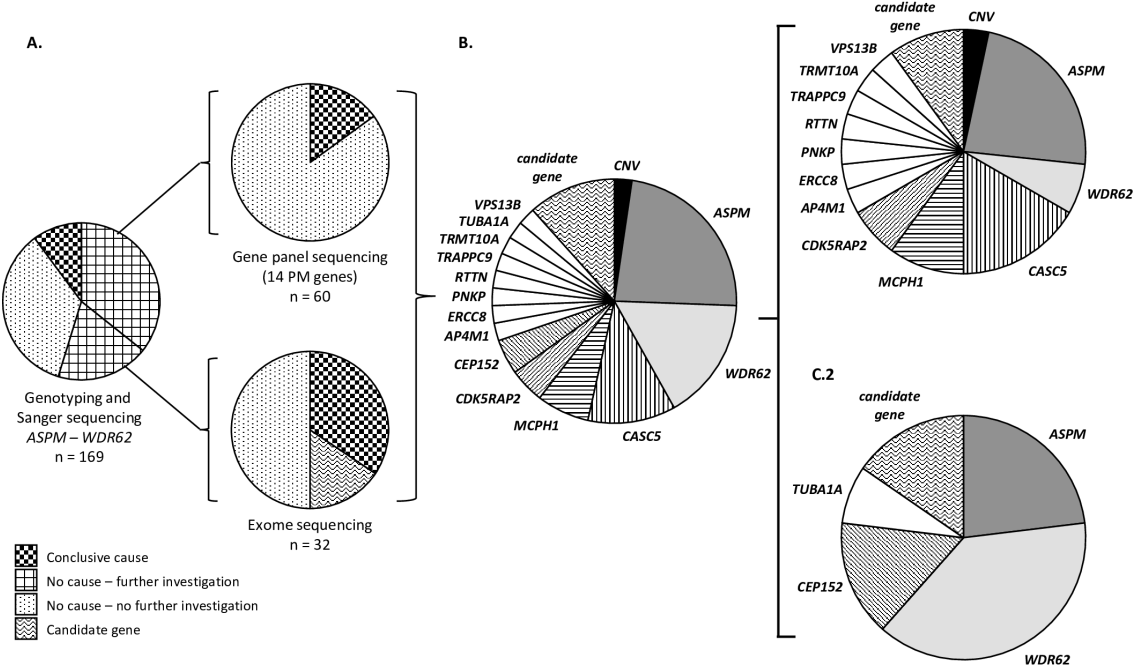


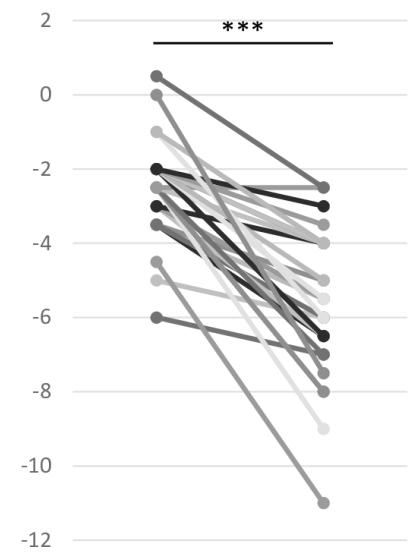

